\title{
Hypomorphic mutations identified in the candidate Leber congenital amaurosis gene CLUAP1
}

\author{
Zachry T. Soens, BS ${ }^{1,2}$, Yuanyuan Li, PhD ${ }^{3}$, Li Zhao, BS ${ }^{2,4}$, \\ Aiden Eblimit, $\mathrm{PhD}^{1,2}$, Rachayata Dharmat, BS ${ }^{1,2}$, Yumei Li, $\mathrm{PhD}^{1,2}$, \\ Yiyun Chen, $\mathrm{PhD}^{1,2}$, Mohammed Naqeeb, MD5 , Norma Fajardo, $\mathrm{PhD}^{6-8}$, \\ Irma Lopez, PhD ${ }^{6-8}$, Zhaoxia Sun, PhD ${ }^{3}$, Robert K. Koenekoop, MD, PhD ${ }^{6-8}$ and Rui Chen, $\mathrm{PhD}^{1,2,4,9,10}$
}

Purpose: Leber congenital amaurosis (LCA) is an early-onset form of retinal degeneration. Six of the 22 known LCA genes encode photoreceptor ciliary proteins. Despite the identification of 22 LCA genes, the genetic basis of $\sim 30 \%$ of LCA patients remains unknown. We sought to investigate the cause of disease in the remaining $30 \%$ by examining cilia-associated genes.

Methods: Whole-exome sequencing was performed on an LCA cohort of 212 unsolved probands previously screened for mutations in known retinal-disease genes. Immunohistochemistry using mouse retinas was used to confirm protein localization and zebrafish were used to perform rescue experiments.

Results: A homozygous nonsynonymous mutation was found in a single proband in CLUAP1, a gene required for ciliogenesis and cilia maintenance. Cluap1 knockout zebrafish exhibit photoreceptor cell death as early as 5 days after fertilization, and rescue experiments revealed that our proband's mutation is significantly hypomorphic.

Conclusion: Consistent with the knowledge that CLUAP1 plays an important role in cilia function and that cilia are critical to photoreceptor function, our results indicate that hypomorphic mutations in CLUAP1 can result in dysfunctional photoreceptors without systemic abnormalities. This is the first report linking mutations in CLUAP1 to human disease and establishes CLUAP1 as a candidate LCA gene.

Genet Med advance online publication 28 January 2016

Key Words: ciliopathy; CLUAP1; early-onset retinal disease; Leber congenital amaurosis (LCA); photoreceptor connecting cilium

\section{INTRODUCTION}

Leber congenital amaurosis (LCA; http://www.omim.org/phenotypicSeries/PS204000) is the most severe form of nonsyndromic inherited retinal disease, with severe visual impairment within the first year of life. LCA is characterized by nystagmus and an extinguished electroretinogram. LCA affects between $1 / 30,000$ and 1/80,000 individuals, differing by population, and accounts for $5 \%$ of all inherited retinal diseases. ${ }^{1}$ As of 2015 , 22 genes have been linked to LCA; however, conventional techniques for molecular diagnosis find mutations in these genes in only $\sim 70 \%$ of LCA patients, highlighting the genetic heterogeneity of LCA..$^{1-5}$ Gene therapy in the retina of human LCA patients has made significant progress in restoring sight, emphasizing the importance of understanding the genetic etiology of every patient's disease to allow access to such technologies. ${ }^{6}$ Genetic counseling based on a patient's molecular diagnosis also provides the opportunity for mutation screening in offspring and spouses. Discovering the cause of disease in the remaining $~ 30 \%$ of LCA patients is therefore critical to enable treatment for this devastating disease.

An absent electroretinogram, a hallmark of LCA, is indicative of malfunctioning photoreceptor cells, which initiate the recorded electrical signal and are responsible for the generation of the a-wave. ${ }^{7}$ Every identified LCA disease gene has a role relevant to the proper functioning of photoreceptor cells. ${ }^{1,8-14}$ Photoreceptors are remarkable cells with a highly specialized morphology designed to facilitate photon detection and transduction of visual information. The most striking feature of photoreceptors cells is the elongated outer segment, which contains stacks of membrane discs that house the transmembrane photosensitive opsin proteins that capture photons and activate the visual transduction cascade. The outer segment is linked to the inner segment of photoreceptor cells by a cilium called the connecting cilium, from which the outer segment develops; the outer segment in conjunction with the connecting cilium is considered a large modified primary cilium. The inner segment contains the

\footnotetext{
${ }^{1}$ Department of Molecular and Human Genetics, Baylor College of Medicine, Houston, Texas, USA; ${ }^{2}$ Human Genome Sequencing Center, Baylor College of Medicine, Houston, Texas, USA; ${ }^{3}$ Department of Genetics, Yale University School of Medicine, New Haven, Connecticut, USA; ${ }^{4}$ Department of Structural and Computational Biology \& Molecular Biophysics, Baylor College of Medicine, Houston, Texas, USA; ${ }^{5}$ Department of Ophthalmology, Um Al Qura University Medical School, Makkah, Saudi Arabia; ${ }^{6}$ McGill Ocular Genetics Laboratory and Centre, Department of Ophthalmology, McGill University Health Centre, Montreal, Quebec, Canada; ${ }^{7}$ Department of Paediatric Surgery, McGill University Health Centre, Montreal, Quebec, Canada; ${ }^{8}$ Department of Human Genetics, McGill University Health Centre, Montreal, Quebec, Canada; ${ }^{9}$ Department of Biochemistry and Molecular Biology, Baylor College of Medicine, Houston, Texas, USA; ${ }^{10}$ Program of Developmental Biology, Baylor College of Medicine, Houston, Texas, USA. Correspondence: Rui Chen (ruichen@bcm.edu)
}

Submitted 30 June 2015; accepted 4 December 2015; advance online publication 28 January 2016. doi:10.1038/gim.2015.205 
cell's protein synthesis machinery; therefore, every protein with a role in the outer segment must be transported through the connecting cilium for proper function. The outer segment undergoes a massive rate of protein turnover to accomplish phototransduction, including $10 \%$ of the outer segment being shed every day. Shed discs are phagocytized by neighboring retinal pigmented epithelial cells and are then replaced through the voluminous protein transport needed for new disc formation. ${ }^{15}$ The connecting cilium is therefore homologous to the transition zone of primary cilia found in other cell types and operates as a gatekeeper of protein trafficking to the outer segment. ${ }^{16}$

Dysfunction of the connecting cilium results in varying degrees of photoreceptor degeneration depending on which component of the connecting cilium is perturbed. In contrast to LCA cilia genes, mutations in $M A K$, which localizes to the connecting cilium, cause a form of retinal degeneration that has its onset at an average age of 20 years. ${ }^{17}$ Highlighting the importance of cilia in retinal disease, $\sim 15 \%$ of known retinal-disease genes are involved in connecting cilium function. Interestingly, this proportion increases to $\sim 25 \%$ when considering only known LCA genes (CEP290, IQCB1, LCA5, RPGRIP1, SPATA7, TULP1), supporting the established notion that cilia are critical to early stages of photoreceptor cell development and maintenance. ${ }^{9,18}$ Combining the knowledge that cilia genes are good retinal-disease gene candidates with the knowledge that cilia are important for early photoreceptor development leads to the conclusion that cilia genes are excellent candidate LCA genes. ${ }^{19}$

Clusterin-associated protein 1 , or CLUAP1, is required for ciliogenesis and localizes to the base and tip of cilia in vitro in mouse embryonic fibroblasts. ${ }^{20}$ CLUAP1 associates with the intraflagellar transport (IFT) complex B group of proteins and undergoes IFT in both invertebrates and vertebrates. ${ }^{21,22}$ Cluap $^{-1-}$ mice exhibit midgestation lethality due to developmental complications attributed to their lack of primary cilia. ${ }^{20}$ Interestingly, zebrafish with abolished cluap1 expression also display premature mortality due to nonexistent ciliogenesis but can survive until at least 11 days postfertilization (dpf), allowing the examination of retinal tissue. Photoreceptor defects are apparent as early as $3 \mathrm{dpf}$, the same time point when wild-type zebrafish photoreceptors are undergoing outer segment formation, and rhodopsin in the mutant fish is mislocalized in the photoreceptor layer, an indication of aberrant IFT. ${ }^{22}$ Aberrant IFT precedes cell death in animal models of LCA caused by mutations in all six known LCA cilia genes. ${ }^{9,23-27}$ The retinas of cluap $^{\text {-1 }}$ zebrafish lack photoreceptor cells by $5 \mathrm{dpf}$, whereas the remaining cell layers are intact. ${ }^{28}$ The CLUAP1 protein contains two major domains, an N-terminal calponin homology-like domain and a C-terminal coiled-coil domain. Both domains are highly conserved from zebrafish to humans, and homologous domains can be found in many microtubule binding proteins. ${ }^{29}$ The two major isoforms of CLUAP1 are expressed in the human retina at moderate levels similar to IFT protein transcripts. ${ }^{30}$

Based on this evidence, we concluded that CLUAP1 is a cilia gene important for photoreceptor outer segment formation in vertebrates and therefore an excellent candidate LCA disease gene. In this study of 212 unsettled LCA patients, we found a single proband homozygous for a nonsynonymous amino acid substitution in CLUAP1. Rescue experiments using zebrafish as a model system proved that our mutant allele is hypomorphic.

\section{MATERIALS AND METHODS \\ Patient recruitment, clinical diagnosis, and DNA preparation}

Proband MOGL3628 was recruited and diagnosed with LCA at the Montreal Children's Hospital at the McGill University Health Centre. This patient was diagnosed with LCA after we found nystagmus and very poor visual fixation at 6 weeks of age. An electroretinogram was nondetectable and the retina appeared normal. There were no systemic abnormalities. This study was approved by the McGill University Health Centre Research Institute Research Ethics Board and adhered to the tenets of the Declaration of Helsinki. Blood samples were collected from the proband and both parents after obtaining informed consent; DNA was extracted using the Qiagen blood genomic DNA extraction kit (Qiagen, Valencia, CA).

\section{Whole-exome sequencing and next-generation sequencing data processing}

One microgram of total patient DNA was sheared into 300- to 500-bp fragments. Fragments were end-repaired and a $3^{\prime}$ adenosine base added. Illumina Y-shaped adapters (Illumina, San Diego, CA) were ligated to DNA fragments, and 10 cycles of PCR were used to amplify the library with a unique barcode. Library DNA concentration was quantified using the Life Technologies picogreen assay (Life Technologies, Carlsbad, CA), and the library was pooled with five other libraries. Three micrograms of pooled DNA were capture-enriched using the NimbleGen SeqCap EZ Human Exome Library v2.0 Hybridization and Wash Kit (NimbleGen, Madison, WI). Exome-captured libraries were quantified and multiplex sequenced on an Illumina HiSeq 2000.

Next-generation sequencing reads were mapped to the hg19 human reference genome using BWA-MEM, duplicate reads were removed using Picard, local realignments were performed using GATK, and variants were called using Atlas2. A population frequency threshold of $0.5 \%$ was used to filter out common variants that occur too frequently to be the cause of a rare Mendelian disease. Four next-generation sequencing cohort databases were used to determine allele frequencies. The functional consequence of the remaining rare variants was annotated using ANNOVAR, and dbNSFP was used to compile in silico predictions about the deleteriousness of nonsynonymous variants. UGENE was used to perform the multiple sequence alignment using the MUSCLE alignment algorithm.

Please see the Supplementary Methods online for references and details.

\section{Sanger sequencing}

Sanger sequencing was used to confirm the authenticity of the variant identified by next-generation sequencing, to confirm the variant properly segregated in the proband's parents, and to 
screen five additional LCA probands for mutations in CLUAP1. A PCR primer pair was designed for each exon of interest. After PCR amplification, the amplicons were sequenced on an Applied Biosystems 3730XL or 3500XL Genetic Analyzer (Applied Biosystems, Foster City, CA).

\section{Immunohistochemistry}

In vivo immunohistochemistry was performed on adult mouse retinal sections. Anti-CLUAP1 and anti-acetylated $\alpha$-tubulin were used for primary antibodies, and 4',6-diamidino-2-phenylindole was used as a counterstain. In vitro immunohistochemistry was performed on hTERT-RPE1 cells transiently transfected to overexpress human FLAG-tagged CLUAP1 under control of a CMV promoter. CLUAP1 cDNA was mutagenized to recreate the proband's mutation. Anti-FLAG tag and antiacetylated $\alpha$-tubulin were used for primary antibodies, and 4',6-diamidino-2-phenylindole was used as a counterstain.

Please see the Supplementary Methods online for experimental details, reagent sources, and reagent concentrations.

\section{Zebrafish functional experiments}

The proband's mutation was recreated at the homologous zebrafish cDNA residue. Capped mRNA was amplified, template DNA was degraded, and mRNA was purified. Embryos were lysed according to a previously published protocol. ${ }^{31}$ Day 0 embryos were lysed at 8-9 hours postfertilization (hpf), and day 1 embryos were lysed at $24 \mathrm{hpf}$. Western blotting was performed using anti-GFP. Anti- $\beta$-tubulin was used as a loading control. Zebrafish rescue experiments were performed by injecting embryos from cluap $^{-1+}$ incrosses at the one-cell stage with varying concentrations of zebrafish wild-type and mutant cluap1 mRNA tagged with GFP. GFP mRNA was injected as a negative control. An average of 96.5 embryos was injected per allele per concentration and for controls. The percentage of phenotypic zebrafish was quantified at $3 \mathrm{dpf}$. Rescue experiments were performed twice; therefore, reported data are the average of two experiments. $P$ values were obtained using a Student $t$-test. Error bars represent the standard deviation of the two independent experiments.

\section{RESULTS}

Whole-exome sequencing identifies homozygous variants in CLUAP1 as candidate pathogenic mutations in an LCA proband

Saudi Arabian proband MOGL3628, currently age 5 years, exhibited severe visual function limited to light perception by 6 weeks of age, accompanied by nystagmus, the oculodigital sign, and an extinguished electroretinogram. The proband's fundi appeared relatively normal (Supplementary Figure S1 online). Whole-exome sequencing (WES) identified 349 rare protein-altering variants (Supplementary Table S1 online). No causal mutations were found among the eight variants located in known retinal-disease genes (Supplementary Table S2 online), so a list of all potentially biallelic variants was created assuming the recessive inheritance pattern usually seen in LCA patients
(Supplementary Table S3 online). Given the importance of cilia-associated genes in retinal disease, a list of cilia genes (Supplementary Methods online) was compared with the genes present in the biallelic variant list, and the cilia gene CLUAP1 was identified as containing a homozygous nonsynonymous mutation that prompted further analysis of this proband. All 35 potentially biallelic variants in 21 genes were subsequently subjected to a systematic candidate disease gene prioritization strategy to exclude or prioritize each gene for further testing. Both genelevel and variant-level information were leveraged during the prioritization process, and the primary reasons for gene exclusion or prioritization are listed in Supplementary Table S3 online. A description of the process is found in the Supplementary Methods. The mutations in CLUAP1 were deemed the most likely candidates for the cause of disease; therefore, their authenticity was validated and proper segregation of the mutations was confirmed by Sanger sequencing (Figure 1a).

CLUAP1 has two isoforms that are both expressed in the human retina: a long isoform (NM_015041,NP_055856), which encodes 413 amino acids, and a short isoform (NM_024793, NP_079069), which encodes 247 amino acids. The mutation occurs in the eighth exon of CLUAP1 and affects the cDNA sequence of both isoforms, c.817C $>\mathrm{T}$ long and c.319C $>\mathrm{T}$ short (Table 1). This mutation results in a leucine-to-phenylalanine substitution, p.L273F long and p.L107F short (Table 1), which is predicted to either be damaging or have a functional impact by $11 / 12$ of the in silico prediction algorithms queried in dbNSFP (Table 1). The mutation is extraordinarily rare-it is found in the Exome Aggregation Consortium database at a frequency of only $1 / 121,086$ chromosomes or $0.0008 \%$, although Saudi Arabians are underrepresented in the examined databases and the single individual seen to be a carrier for the allele is of reported European descent (Table 1).

Our proband's mutation falls in CLUAP1's coiled-coil domain, one of two characterized functional domains in the CLUAP1 protein. Both the coiled-coil and calponin homologylike domains have been reported to be involved in microtubule binding activity, as shown in IFT proteins with homologous domains. The coiled-coil domain is also associated with facilitating protein-protein interactions, and it is within this region that our proband's mutation lies (Figure 1b). A protein multiple sequence alignment of human CLUAP1 and its orthologs reveals that the affected leucine residue is conserved in all sighted organisms, including the fruit fly Drosophila melanogaster and the body louse Pediculus humanus corporis, whose protein sequences display much less overall conservation compared with analyzed vertebrates. Like CLUAP1, the worm ortholog DYF-3 has been shown to be required for neuronal sensory cilium formation in Caenorhabditis elegans, but the specific residue is not conserved in either worm aligned (Figure 1c). ${ }^{32}$

To ascertain additional patients with CLUAP1 mutations, we corresponded with our LCA expert clinician-scientist collaborator (R.K.K.), who was able to identify five additional unsolved LCA probands for whom homozygosity mapping 
a
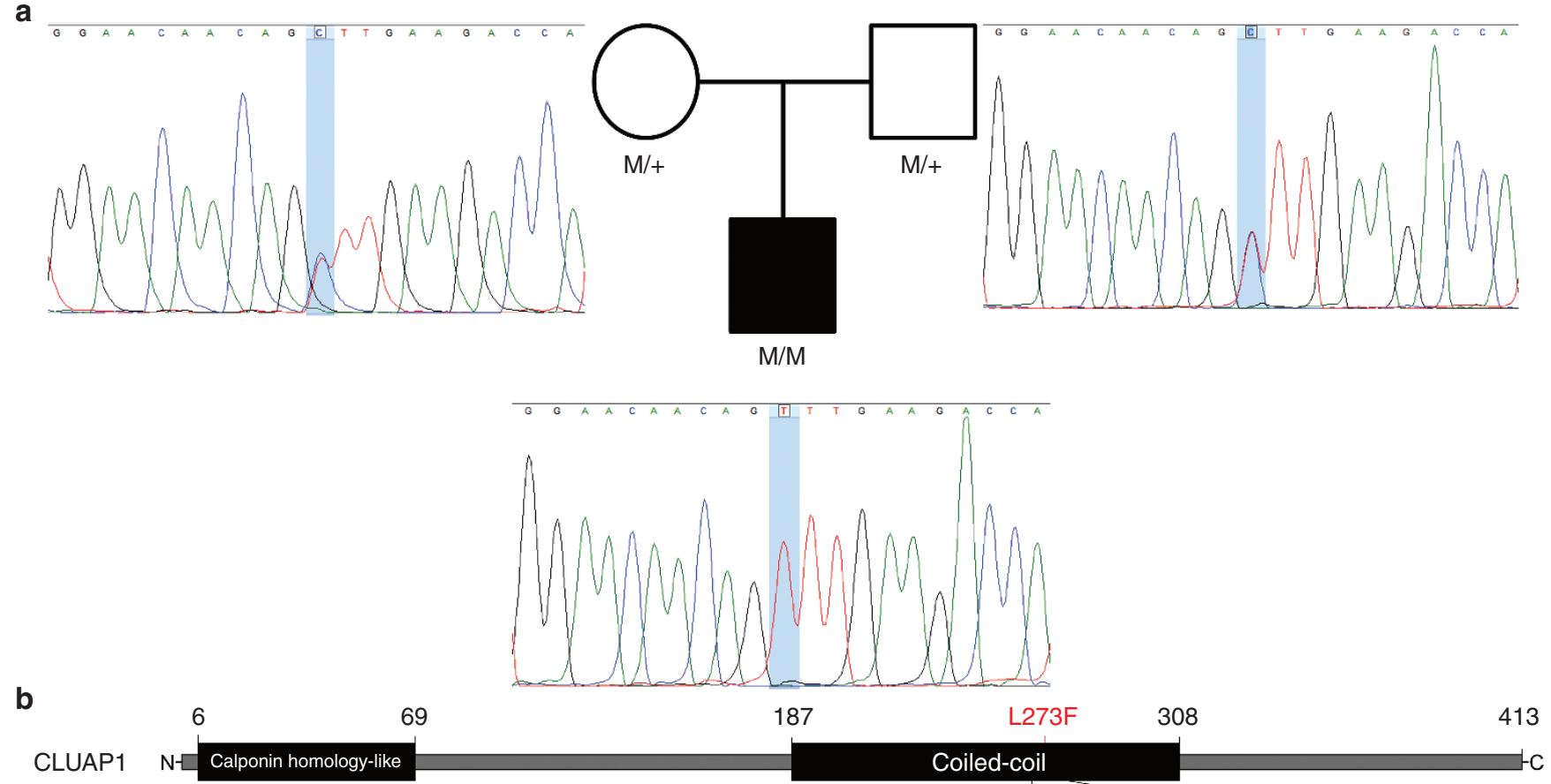

CLUAP1 N-Calponin homology-like

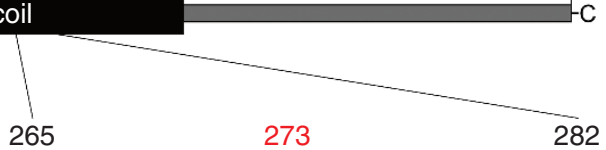

gi|52345626|ref|NP_055856.1| clusterin-associated protein 1 isoform 1 [Homo sapiens] gi|525345411|ref|NP_001267281.1| clusterin-associated protein 1 [Pan troglodytes] gi|548961985|ref|NP_001272094.1| clusterin-associated protein 1 [Macaca fascicularis] gi|62079185|ref|NP_001014247.1| clusterin-associated protein 1 [Rattus norvegicus] gi|39930457|ref|NP_084014.1| clusterin-associated protein 1 [Mus musculus] gi|350529391|ref|NP_001231929.1| clusterin-associated protein 1 [Sus scrofa] gi|157428024|ref|NP_001098920.1| clusterin-associated protein 1 [Bos taurus] gi|478431065|ref|NP_001264520.1| clusterin-associated protein 1 [Gallus gallus] gi|47087642|ref|NP_998173.1| clusterin-associated protein 1 homolog [Danio rerio] gi|148227036|ref|NP_001090466.1| clusterin-associated protein 1 [Xenopus laevis] gi|62858885|ref|NP_001016969.1| clusterin-associated protein 1 [Xenopus (Silurana) tropicalis] gi|24643822|ref|NP_608470.1| CG17599 [Drosophila melanogaster] gi|242017349|ref|XP_002429152.1| Clusterin-associated protein, putative [Pediculus humanus corporis] gi|268553493|ref|XP_002634732.1| C. briggsae CBR-DYF-3 protein [Caenorhabditis briggsae] gi|71981971|ref|NP_001021294.1| Protein DYF-3, isoform a [Caenorhabditis elegans]

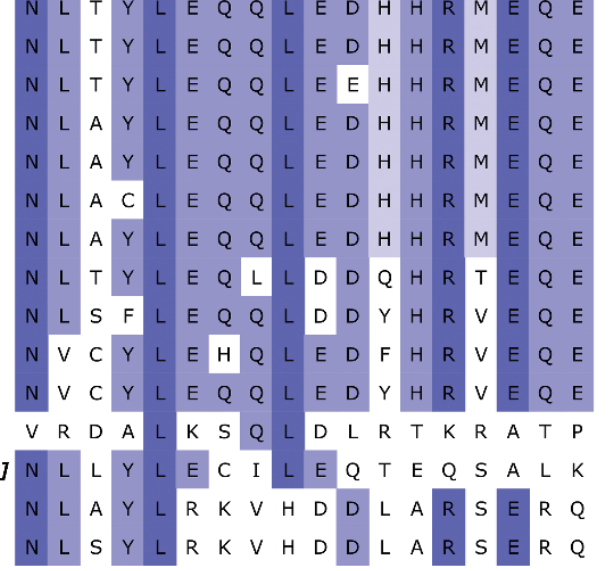

Figure 1 Pedigree, Sanger sequencing traces, and 2D CLUAP1 protein structure with an multiple sequence alignment of the affected residue. (a) The proband's pedigree and Sanger sequencing traces showing proper segregation of the mutant allele. (b) A 2D graphical representation of the long isoform of CLUAP1 containing two characterized functional domains with the location of the proband's mutation displayed in red. A protein multiple sequence alignment surrounding the amino acid affected by the proband's mutation showing the leucine at position 273 is conserved in sighted organisms. Aligned columns are colored a darker blue with increasing conservation at that position.

had mapped a significant homozygous interval surrounding and containing CLUAP1. Direct Sanger sequencing of the CLUAP1 gene in these individuals did not identify any candidate mutations. We additionally contacted members of the European Retinal Disease Consortium, of which R.K.K. is a member, and indicated our interest in CLUAP1 via GeneMatcher, a component of the Matchmaker Exchange network, but we were unable to identify potential collaborators who had retinal-disease patients with CLUAP1 mutations.
CLUAP1 is localized at the connecting cilium of photoreceptor cells

Although CLUAP1 is proposed to be a ciliary protein based on functional studies, its precise localization in the retina is unknown. Given our proband's phenotype, we hypothesized that CLUAP1 is likely to be expressed in photoreceptor cells. To test this, we performed immunohistochemistry on sections of mouse retinas using an anti-CLUAP1 antibody as well as anti-acetylated $\alpha$-tubulin and 4',6-diamidino-2-phenylindole. CLUAP1 localizes to the connecting cilium of photoreceptor 
cells located between the inner and outer segment layers of the retina (Figure 2). An inconsistent CLUAP1 signal is also seen in certain areas of the outer nuclear layer, but this may be the result of nonspecific antibody binding.

\section{The proband's missense mutation negatively impacts CLUAP1 function}

To investigate whether the proband's mutation affects CLUAP1 function by altering its localization, we performed immunofluorescence on hTERT-RPE1 cells transiently overexpressing either FLAG-CLUAP1 or FLAG-CLUAP1 ${ }^{\text {Mut }}$. CLUAP1 harboring the proband's mutation was observed to localize to the base of cilia in a manner similar to that of the wild type (Supplementary Figure S2 online). To determine whether the proband's mutation is detrimental to CLUAP1 function in vivo, mutagenesis of wild-type zebrafish cluap1 cDNA was performed to generate a cDNA construct containing a mutation at the zebrafish residue that is homologous to the human variant. Taking advantage of the cluap1 knockout zebrafish model, which shows developmental defects typical for a ciliopathy, a functional analysis of the mutant cluap1 was performed using rescue experiments. As described in the Materials and Methods section, GFP-tagged wild-type and mutant mRNA were injected into zebrafish embryos resulting from cluap $^{+/-}$incrosses.

To investigate whether the mutation affects cluap1 expression, we first performed western blots using embryo lysates. Mutant Cluap1 was expressed at levels similar to those of wild type, leading to the conclusion that the mutation does not affect protein expression or degradation (Figure 3a). To assess the dysfunction of our mutant Cluap1, we screened for the ventral spine-curvature feature present in cluap $^{-/-}$knockout zebrafish at an early age. The percentage of phenotypic embryos was compared with the expected $\sim 25 \%$ observed after control injections of GFP. To determine whether mutant Cluap1 could rescue the phenotype of cluap $^{-/-}$knockout zebrafish, low and high concentrations ( 1 and $130 \mathrm{pg} / \mathrm{nl}$ ) of mutant mRNA were tested along with those of wild type. At the lower concentration of $1 \mathrm{pg} / \mathrm{nl}$, no significant rescue effect was observed from mutant cluap1 mRNA-injected embryos (25.7\% phenotypic progeny, $P=0.118$, Figure $3 \mathrm{~b}$ and Supplementary Table S4), whereas wild-type cluap1 mRNA displayed a borderline significant rescue (9.1\% phenotypic progeny, $P=0.053$, Figure $3 b$ and Supplementary Table S4). At the higher concentration of $130 \mathrm{pg} / \mathrm{nl}$, mutant cluap1 performed similarly to wild type and

\section{Table 1 Mutation information}

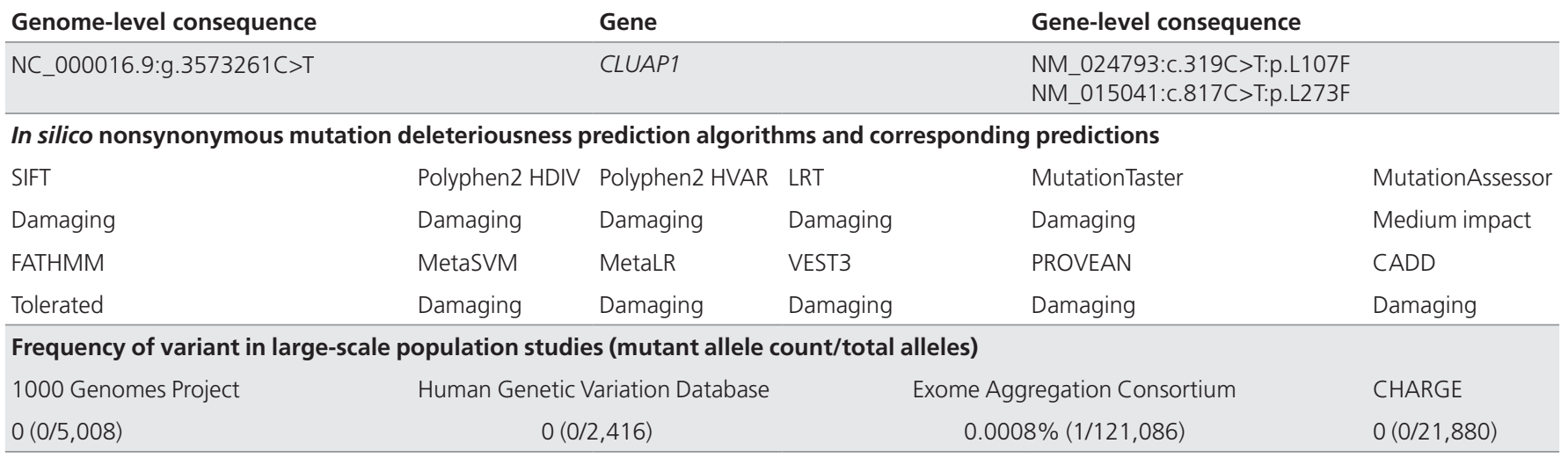

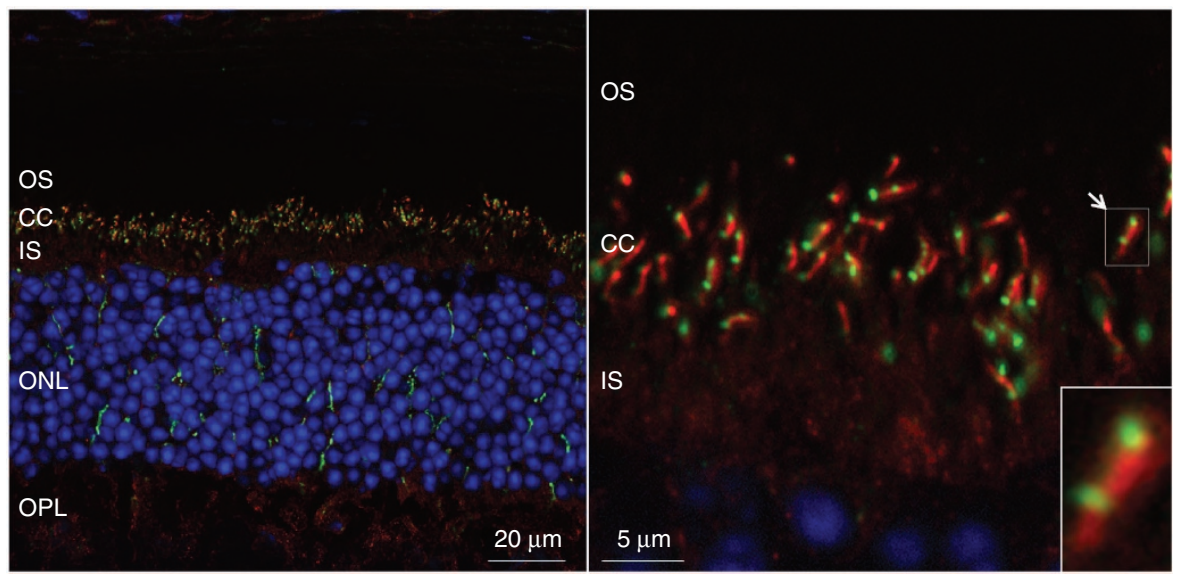

Figure 2 CLUAP1 localizes to the connecting cilium in adult mouse retinas. Mouse immunohistochemistry staining using anti-CLUAP1 (green), antiacetylated $\alpha$-tubulin (red), and 4',6-diamidino-2-phenylindole (blue). CLUAP1 can be seen to localize to specific puncti between the inner segment (IS) and outer segment (OS) layers of photoreceptors cells. CLUAP1 localization overlaps with the tip and base of the acetylated $\alpha$-tubulin staining corresponding to the tip and base of connecting cilia (CC). ONL, outer nuclear layer; OPL, outer plexiform layer. 


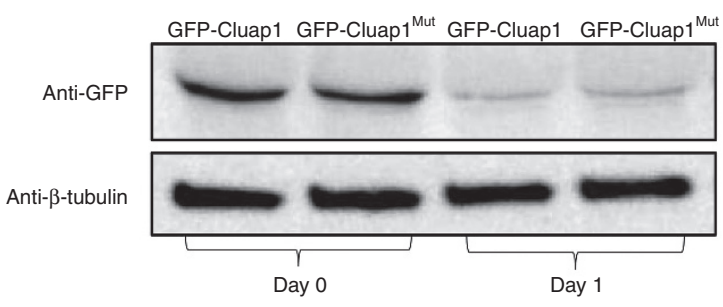

b

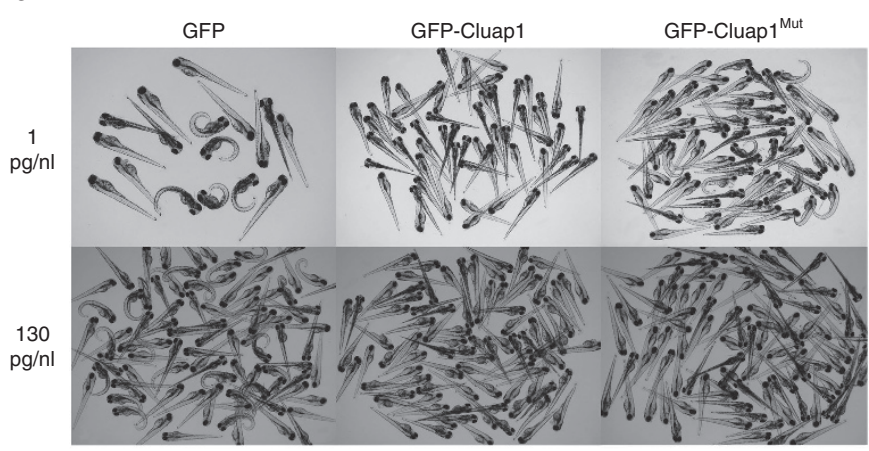

C

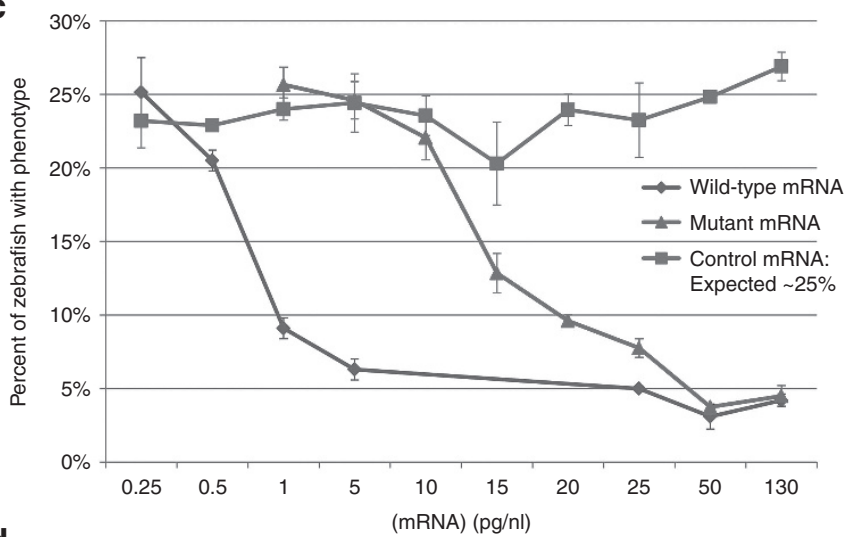

d

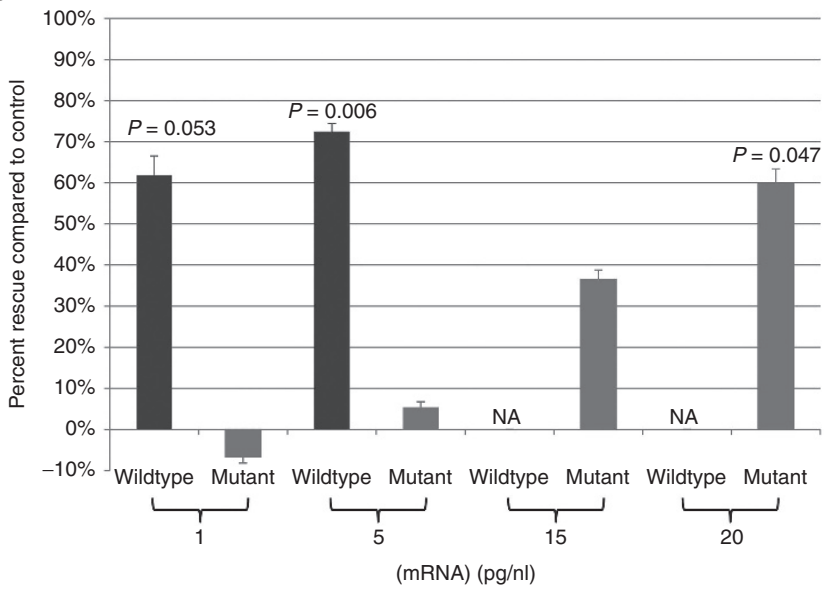

Figure 3 Mutant Cluap1 is expressed at levels similar to those of wild type but requires 20 times the amount to achieve an equivalent rescue effect. (a) Zebrafish embryos injected with $25 \mathrm{pg} / \mathrm{nl}$ GFP-cluap1 or GFP-cluap 1 ${ }^{\text {Mut }}$ mRNA express similar amounts of Cluap1 as verified by western blotting. (b) Embryos resulting from cluap $1^{1 / 4}$ incrosses injected with control GFP mRNA exhibit the expected $25 \%$ phenotypic proportion. At a low concentration (1 $\mathrm{pg} / \mathrm{nl})$, wild-type cluap1 mRNA noticeably rescues the spine-curvature feature of cluap $1^{-1-}$ zebrafish embryos, whereas mutant cluap1 mRNA does not. At a high concentration $(130 \mathrm{pg} / \mathrm{hl})$, mutant cluap $1 \mathrm{mRNA}$ rescues the spine-curvature feature comparable to wild-type cluap 1 mRNA, revealing a dose-dependent response. (c) Dose-response curves of rescue experiments. Zebrafish injected with control GFP mRNA (boxes) showed approximately the expected 25\% Mendelian ratio. Zebrafish injected with GFP-cluap1 mRNA (circles) reached a borderline significant $(P=0.053)$ rescue at $1 \mathrm{pg} / \mathrm{nl}$. Zebrafish injected with GFPcluap $1^{\text {Mut }}$ mRNA (triangles) significantly $(P=0.047)$ rescued the mutant phenotype at a concentration of $20 \mathrm{pg} / \mathrm{hl}$. (d) The percentage of progeny rescued as compared with control injections at the same concentration highlights the 20-fold increase in the amount of mutant cluap1 mRNA required to achieve a level of rescue significance comparable to wild-type cluap1. Errors bars in $\mathbf{c}$ and $\mathbf{d}$ represent the SD from two independent experiments.

exhibited a clearly significant rescue effect (4.5\% phenotypic progeny, $P=0.007$, Figure $3 \mathrm{~b}$ and Supplementary Table $\mathbf{S 4}$ ).

Because a high concentration of mutant cluap1 rescued the spine-curvature feature, we next quantified the functionality of the mutant protein by varying the concentration of cluap1 mRNA injections from 0.25 to $130 \mathrm{pg} / \mathrm{nl}$. Graphing the doseresponse curve of these rescue experiments revealed that the proband's mutation does indeed interfere with Cluap1 function. Although wild-type cluap1 mRNA can reach a borderline significant rescue effect at the previously mentioned concentration of $1 \mathrm{pg} / \mathrm{nl}$, mutant cluap $1 \mathrm{mRNA}$ requires a concentration of $20 \mathrm{pg} / \mathrm{nl}$ to achieve a similar level of rescue significance $(9.6 \%$ phenotypic progeny, $P=0.047$, Figure $3 \mathrm{c}$ and Supplementary Table S4 online). To visualize these data in an alternative form, we compared the percentage of progeny expected to be phenotypic based on control injections to the percentage actually observed after mutant and wild-type cluap1 mRNA injections to determine the percentage of progeny that were rescued, which highlights the hypomorphic nature of our proband's allele (Figure 3d). Based on these functional rescue assays, Cluap1 containing our proband's mutation has as little as $5 \%$ of its remaining activity, as judged from the 20 -fold increase in mutant Cluap1 concentration required to achieve a rescue significance comparable to that of wild type.

\section{DISCUSSION}

Because CLUAP1 is required for photoreceptor function in other vertebrates, and because our LCA proband's allele is an extremely hypomorphic CLUAP1 allele, we conclude that CLUAP1 is very likely to be necessary for photoreceptor function in humans. Therefore, this is the first report linking hypomorphic mutations in CLUAP1 to human disease and establishes CLUAP1 as a candidate LCA gene. Identifying CLUAP1 as a novel candidate LCA gene is important both for understanding 


\section{ORIGINAL RESEARCH ARTICLE}

human biology and for unlocking the future molecular diagnosis of patients affected by mutations in this gene. CLUAP1 can now be studied in the context of being a human ciliopathy gene, and establishing a new candidate disease gene allows for faster and less expensive molecular diagnosis of future patients because geneticists can target the CLUAP1 locus for examination. CLUAP1 mutations appear to be a rare cause of LCA, so further studies screening additional patient cohorts are needed to determine the true proportion of LCA patients with CLUAP1 mutations.

The importance of cilia in many areas of the body and the importance of CLUAP1 in cilia raise concerns about the clinical presentation of the presented proband and why syndromic features have not been observed. We considered addressing this question by sectioning the retinas of embryos rescued with mutant cluap 1 mRNA to verify that a retinal phenotype persists after the correction of spine curvature. Unfortunately, given the rapid decay of the injected mRNA and the exogenous protein being minimally detectable at $1 \mathrm{dpf}$ (Figure 3a), it is unlikely that this approach would be informative because photoreceptor development is not complete until around $5 \mathrm{dpf}$. However, three possible explanations exist for this observation. The first is that our proband does indeed have a syndromic disease but additional features have yet to manifest due to the young age. This would not be surprising because multiple syndromic ciliopathies such as Joubert syndrome, Senior-Løken syndrome, and Alström syndrome are known to present a retinal-degeneration feature preceding complications in other tissues. These syndromes can in fact be misdiagnosed as LCA because of the lack of additional symptoms in an affected individual's initial clinical presentation. ${ }^{1}$

The second explanation is that the specific mutation that our proband harbors is detrimental only to a retina-specific function and therefore only a retinal phenotype is observed. The connecting cilium in photoreceptors is a unique form of cilia containing proteins not found in most other ciliated cell types in the body. The presence of retina-specific proteins at the connecting cilium means that proteins like CLUAP1 whose expression is not restricted to the retina conceivably have a purpose specialized to the connecting cilium involving interactions with retina-specific proteins. This explanation is supported by the fact that the proband's mutation causes a nonsynonymous amino acid substitution and that the mutation resides in CLUAP1's coiled-coil domain known to accommodate protein-protein interactions. The residue that our proband's mutation affects is not conserved in Caenorhabditis elegans and Caenorhabditis briggsae, implying that the residue is not essential for ciliogenesis but instead is important for some function specific to the organisms in which it is strictly conserved. The absence of eyes is one of the defining features separating these worms from the other species in the multiple sequence alignment; therefore, a safe hypothesis appears to be that the conserved leucine is required for a function involved in vision.
The third explanation is that our proband's mutation results in a general hypomorphic form of CLUAP1 and that the retina is more sensitive to this decrease in CLUAP1 function than other tissue types, possibly comparable to the ubiquitously expressed LCA gene NMNAT1. ${ }^{8}$ The variability in phenotype caused by mutations in the same gene can range drastically, especially in retinal disease, depending on the exact nature of the patients' alleles. It is possible that a mutation residing in CLUAP1's calponin homology-like domain would confer a different phenotype impacting other tissues, and it is likely that the complete loss of function of CLUAP1 would not be compatible with human life as seen in other vertebrates. The function and expression of genes are also not always conserved across species, and human CLUAP1 is not detected in the human kidney by northern blotting, which could explain the lack of kidney defects observed in the original zebrafish polycystic kidney disease screen. ${ }^{33,34}$

In 2008, known LCA genes were reported to account for $70 \%$ of LCA cases; based on our experience in 2015, this percentage can vary up to $75 \%$, but for the most part it has changed unappreciatively despite the identification of an additional eight genes. At least six of the reported LCA disease genes, including the two most recently published genes, account for a remarkably small portion ( 1\%) of all LCA cases. ${ }^{11-13,35-37}$ CLUAP1 adds to this trend, accounting for 1/212 LCA patients in our cohort $(0.47 \%)$. More than one-quarter of all LCA patients are waiting for molecular diagnoses, and these patients inspire two interesting hypotheses regarding future directions for studying the genetics of LCA. The first is the big-fish hypothesis, which can be summarized as saying that any one gene responsible for a significant proportion of patients' disease (a "big fish") has probably already been discovered at this stage of human genetics research. This means that only "little fish" are left, which is why we see new LCA disease genes accounting for a minor fraction of patients. It is therefore possible that the source of disease representing the $30 \%$ of unsolved LCA cases will comprise more than 25 novel disease genes, with each accounting for an ever smaller fraction of patients.

The second alternative hypothesis is that the cause of disease resides in known LCA genes but modern genotyping techniques cannot identify the causal variation. For most genetic disorders WES is widely considered the standard method for molecular diagnosis, but it is currently unsuitable for identifying potentially causative variations in introns, promoters, enhancers, and large structural variations. Even in regions captured by WES there are data that we do not fully know how to interpret, including variation in untranslated regions, synonymous variation, and intronic variation close to an exon but outside the 2-bp splicing consensus sequence. Understanding variation in these atypical regions is imperative in the research of human disease because groups may focus efforts on finding novel disease genes when the diseasecausing mutations they seek are "right under their noses." The truth is most likely a combination of these two hypotheses, leaving numerous possibilities for the future of LCA research. 


\section{SUPPLEMENTARY MATERIAL}

Supplementary material is linked to the online version of the paper at http://www.nature.com/gim

\section{ACKNOWLEDGMENTS}

We thank the proband and their family for participating in this study. This work is supported by the National Institutes of Health (grants R01-DK092808 and 1P30-DK090744 to Z. Sun and shared grant 1S10RR026550 to R.C.), the National Eye Institute (grants R01EY022356, R01EY018571, and P30EY002520 to R.C.), the Retina Research Foundation, and the Foundation Fighting Blindness (grant BR-GE-0613-0618-BCM to R.C.). R.K.K. acknowledges the Foundation Fighting Blindness (Canada), the Canadian Institutes of Health Research, and Fonds de Recherché Santé Quebéc et Réseau Vision, all of which supported this work. Z. Soens is supported by National Eye Institute training grant 5T32EY007001-38.

\section{DISCLOSURE}

The authors declare no conflict of interest.

\section{REFERENCES}

1. den Hollander Al, Roepman R, Koenekoop RK, Cremers FP. Leber congenital amaurosis: genes, proteins and disease mechanisms. Prog Retin Eye Res 2008;27:391-419.

2. RetNet Retinal Information Network. University of Texas-Houston Health Science Center, Houston, TX. http://www.sph.uth.tmc.edu/RetNet. Accessed 10 February 2015.

3. Wang X, Wang H, Sun V, et al. Comprehensive molecular diagnosis of 179 Leber congenital amaurosis and juvenile retinitis pigmentosa patients by targeted next generation sequencing. J Med Genet 2013;50:674-688.

4. Wang $X$, Wang $H_{\text {, }}$ Cao $M$, et al. Whole-exome sequencing identifies ALMS1, IQCB1, CNGA3, and MYO7A mutations in patients with Leber congenital amaurosis. Hum Mutat 2011;32:1450-1459.

5. Guo Y, Prokudin I, Yu C, et al. Advantage of whole exome sequencing over allele-specific and targeted segment sequencing, in detection of novel TULP1 mutation in Leber congenital amaurosis. Ophthal Genet 2015;36:333-338.

6. Hufnagel RB, Ahmed ZM, Corrêa ZM, Sisk RA. Gene therapy for Leber congenital amaurosis: advances and future directions. Graefes Arch Clin Exp Ophthalmol 2012;250:1117-1128.

7. Robson JG, Frishman $\mathrm{L}$. The rod-driven a-wave of the dark-adapted mammalian electroretinogram. Prog Retin Eye Res 2014;39:1-22.

8. Koenekoop RK, Wang H, Majewski J, et al.; Finding of Rare Disease Genes (FORGE) Canada Consortium. Mutations in NMNAT1 cause Leber congenital amaurosis and identify a new disease pathway for retinal degeneration. Nat Genet 2012;44:1035-1039.

9. Eblimit A, Nguyen TM, Chen Y, et al. Spata7 is a retinal ciliopathy gene critical for correct RPGRIP1 localization and protein trafficking in the retina. Hum Mol Genet 24:1584-1601.

10. Aldahmesh MA, Al-Owain M, Alqahtani F, Hazzaa S, Alkuraya FS. A null mutation in CABP4 causes Leber's congenital amaurosis-like phenotype. Mol Vis 2010;16:207-212.

11. Sergouniotis PI, Davidson AE, Mackay DS, et al. Recessive mutations in KCNJ13, encoding an inwardly rectifying potassium channel subunit, cause leber congenital amaurosis. Am J Hum Genet 2011;89:183-190.

12. Henderson RH, Williamson $\mathrm{KA}$, Kennedy JS, et al. A rare de novo nonsense mutation in OTX2 causes early onset retinal dystrophy and pituitary dysfunction. Mol Vis 2009;15:2442-2447.

13. Asai-Coakwell M, March L, Dai XH, et al. Contribution of growth differentiation factor 6-dependent cell survival to early-onset retinal dystrophies. Hum Mol Genet 2013;22:1432-1442.

14. Estrada-Cuzcano A, Koenekoop RK, Coppieters F, et al. IQCB1 mutations in patients with leber congenital amaurosis. Invest Ophthalmol Vis SCi 2011;52:834-839.
15. Nguyen-Legros J, Hicks D. Renewal of photoreceptor outer segments and their phagocytosis by the retinal pigment epithelium. Int Rev Cytol 2000;196: 245-313.

16. Wheway G, Parry DA, Johnson CA. The role of primary cilia in the development and disease of the retina. Organogenesis 2014;10:69-85.

17. van Huet RA, Siemiatkowska AM, Özgül RK, et al. Retinitis pigmentosa caused by mutations in the ciliary MAK gene is relatively mild and is not associated with apparent extra-ocular features. Acta Ophthalmol 2015;93:83-94.

18. Rachel RA, Li T, Swaroop A. Photoreceptor sensory cilia and ciliopathies: focus on CEP290, RPGR and their interacting proteins. Cilia 2012;1:22.

19. Liu Q, Zhang Q, Pierce EA. Photoreceptor sensory cilia and inherited retinal degeneration. Adv Exp Med Bio/ 2010;664:223-232.

20. Botilde Y, Yoshiba S, Shinohara K, et al. Cluap1 localizes preferentially to the base and tip of cilia and is required for ciliogenesis in the mouse embryo. Dev Biol 2013;381:203-212.

21. Omori Y, Zhao C, Saras A, et al. Elipsa is an early determinant of ciliogenesis that links the IFT particle to membrane-associated small GTPase Rab8. Nat Cell Biol 2008:10:437-444.

22. Lee C, Wallingford JB, Gross JM. Cluap1 is essential for ciliogenesis and photoreceptor maintenance in the vertebrate eye. Invest Ophthalmol Vis Sci 2014;55:4585-4592.

23. Boldt K, Mans DA, Won J, et al. Disruption of intraflagellar protein transport in photoreceptor cilia causes Leber congenital amaurosis in humans and mice. J Clin Invest 2011;121:2169-2180.

24. Anand M, Khanna H. Ciliary transition zone (TZ) proteins RPGR and CEP290: role in photoreceptor cilia and degenerative diseases. Expert Opin Ther Targets 2012;16:541-551.

25. Ronquillo CC, Bernstein PS, Baehr W. Senior-Løken syndrome: a syndromic form of retinal dystrophy associated with nephronophthisis. Vision Res 2012;75: 88-97.

26. Grossman GH, Watson RF, Pauer GJ, Bollinger K, Hagstrom SA. Immunocytochemical evidence of Tulp1-dependent outer segment protein transport pathways in photoreceptor cells. Exp Eye Res 2011;93:658-668.

27. Won J, Gifford E, Smith RS, et al. RPGRIP1 is essential for normal rod photoreceptor outer segment elaboration and morphogenesis. Hum Mol Genet 2009; 18:4329-4339

28. Li J, Sun Z. Qilin is essential for cilia assembly and normal kidney development in zebrafish. PLoS One 2011;6:e27365.

29. Schou KB, Andersen JS, Pedersen LB. A divergent calponin homology (NN-CH) domain defines a novel family: implications for evolution of ciliary IFT complex B proteins. Bioinformatics 2014;30:899-902.

30. Farkas MH, Grant GR, White JA, Sousa ME, Consugar MB, Pierce EA. Transcriptome analyses of the human retina identify unprecedented transcript diversity and $3.5 \mathrm{Mb}$ of novel transcribed sequence via significant alternative splicing and novel genes. BMC Genomics 2013;14:486.

31. Zamora LY, Lu Z. Alcohol-induced morphological deficits in the development of octavolateral organs of the zebrafish (Danio rerio). Zebrafish 2013;10: $52-61$.

32. Murayama T, Toh Y, Ohshima Y, Koga M. The dyf-3 gene encodes a novel protein required for sensory cilium formation in Caenorhabditis elegans. $J \mathrm{Mol}$ Biol 2005;346:677-687.

33. Takahashi M, Lin YM, Nakamura Y, Furukawa Y. Isolation and characterization of a novel gene CLUAP1 whose expression is frequently upregulated in colon cancer. Oncogene 2004;23:9289-9294.

34. Sun Z, Amsterdam A, Pazour GJ, Cole DG, Miller MS, Hopkins N. A genetic screen in zebrafish identifies cilia genes as a principal cause of cystic kidney. Development 2004;131:4085-4093.

35. Abu-Safieh L, Alrashed M, Anazi S, et al. Autozygome-guided exome sequencing in retinal dystrophy patients reveals pathogenetic mutations and novel candidate disease genes. Genome Res 2013;23: 236-247.

36. Thompson DA, Li Y, McHenry CL, et al. Mutations in the gene encoding lecithin retinol acyltransferase are associated with early-onset severe retinal dystrophy. Nat Genet 2001;28:123-124.

37. Mackay DS, Henderson RH, Sergouniotis PI, et al. Novel mutations in MERTK associated with childhood onset rod-cone dystrophy. Mol Vis 2010;16: 369-377. 